\title{
Trend Analysis of Three Major Mosquito Borne Diseases in Punjab, India
}

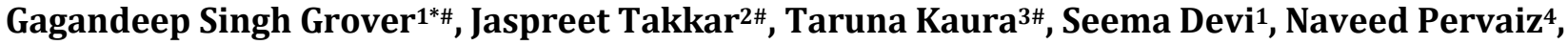 Upninder Kaur ${ }^{3}$, Rakesh Sehgal ${ }^{3}$}

\author{
${ }^{1}$ National Vector Borne Disease Control Programme, Chandigarh, Punjab, India \\ ${ }^{2}$ Department of Physiology, Gian Sagar Medical College, Punjab, India \\ ${ }^{3}$ Department of Medical Parasitology, PGIMER, Chandigarh, India \\ ${ }^{4}$ Department of Zoology, Panjab University, Chandigarh, India \\ Email: `dr.gagangrover@gmail.com, jaspreettkkr77@gmail.com, kaurataruna@gmail.com,minturam26@gmail.com, \\ naveedpervaiz999@gmail.com,drupninderkaur@gmail.com, sehgalpgi@gmail.com
}

How to cite this paper: Grover, G.S., Takkar, J., Kaura, T., Devi, S., Pervaiz, N., Kaur, U. and Sehgal, R. (2020) Trend Analysis of Three Major Mosquito Borne Diseases in Punjab, India. Journal of Biosciences and Medicines, 8, 1-11.

https://doi.org/10.4236/jbm.2020.85001

Received: March 19, 2020

Accepted: April 27, 2020

Published: April 30, 2020

Copyright () 2020 by author(s) and Scientific Research Publishing Inc. This work is licensed under the Creative Commons Attribution International License (CC BY 4.0).

http://creativecommons.org/licenses/by/4.0/

\begin{abstract}
Background \& Objectives: Understanding the distribution and prevalence of three major mosquito borne diseases in an area is critical for the development of effective vector control strategies and public health interventions. The present study is therefore aimed to explore the epidemiological trend of malaria, dengue and chikungunya from 2012 to 2018 in Punjab. Methods: Quantitatively retrospective data was collected from Department of Health and Family Welfare, the National Vector Borne Disease Control Programme (NVBDCP), Punjab from 2012 to March 2018. The collected data was statistically analysed. Results: In case of malaria highest prevalent districts in Punjab are Mansa and Bathinda, for dengue Patiala, Ludhiana and S.A.S. Nagar and Patiala for chikungunya. Malaria was reported mainly from rural areas while dengue and chikungunya were found more in urban areas. For all three mosquito borne diseases, males were infected more as compared to females. Malaria cases were reported in months of August and September while dengue cases increased from July to November whereas chikungunya cases were highest in months of $\mathrm{Au}$ gust to October. Conclusions: These findings help in concluding the trend analysis which in turn helps us to increase our focus on disease endemic districts along with boosting the vector control strategies in respective districts. Further the control of these mosquito borne diseases can be solved by employing adequate human resources, by increasing awareness in the community by conducting health camps and strengthening the entomological surveillance for timely reduction in the breeding of the vectors, especially before the repeated rise in cases during the period from July to November each year.
\end{abstract}

"Consider first three authors as joint first author. 


\section{Keywords}

NVBDCP, Malaria, Dengue, Chikungunya

\section{Introduction}

The vector borne diseases (VBDs) contribute around 17\% of the estimated global burden of all human infectious diseases and recently the World Health Organization has reported that the number of deaths occurring annually due to VBDs is 70,000 and more than 3.9 billion people are at risk of infection [1]. Malaria, Dengue and Chikungunya, etc. are some of the most common vector borne diseases in the world and are one of the major health problems in India. Malaria and dengue constitute the most deadly and the world's fastest spreading vector-borne diseases though the incidence of malaria cases has been observed to decrease over the last five years worldwide by over $20 \%$ and the mortality rate has been decreased by $30 \%$ [1]. Moreover, India has also experienced drastic decrease in malaria cases and has formulated National Framework for Malaria Elimination (2016-2030) in close alignment with the Global Technical Strategy for Malaria, Roll Back Malaria Action (RBM). In Punjab state (India), malaria is declining except in some districts where the malaria burden has remained unchanged or increased. Thus, more extensive and exact data on the actual malaria burden will be important to guide control on the path to achieve malaria elimination.

In addition to malaria, dengue, an arboviral infection is also a major public health concern as the prevalence of it has increased and spread in recent decades in tropical and subtropical regions. It has been reported that more than half of the world's population is at risk of dengue infection and $34 \%$ of global cases are from India only [1] [2]. Chikungunya, another mosquito transmitted disease caused by chikungunya virus (CHIKV) is also of great public health concern in India. After 1973, this virus had almost disappeared from India and no case was reported till end of 2005 [3]. The virus then re-emerged in 2005 after a gap of 32 years and an explosive outbreak was reported which affected 13 states in India [4]. The number of cases reported has increased in recent years, particularly during 2016-2017. In 2016, 64,057 cases of CHIK were reported from India only.

The National Vector Borne Disease Control Programme (NVBDCP) is an aegis which includes the programmes for prevention and control of vector borne diseases viz., malaria, filaria, Japanese encephalitis (JE), Dengue/DHF, chikungunya, lymphatic filariasis and Kala Azar. The programme is executed as per the National Policy of Government of India.

Punjab State has semi-urban areas, rich irrigated lands and good monsoon conditions that facilitate prolific growth of mosquitoes and transmission of malaria, dengue and chikungunya. The state is divided into 22 districts. The state of Punjab is also committed to elimination of malaria by 2022 in the line with national goal of elimination. 
Due to growing population, unplanned urbanization, lack of awareness about the diseases and increasing number of slums in urban areas, the incidence of mosquito borne diseases is increasing simultaneously. As there are no published studies so far on the trend of these three major mosquito borne diseases from Punjab, the present study was therefore planned with the objective to evaluate the exact disease burden of malaria, dengue and chikungunya in different districts of Punjab State, India.

\section{Materials and Methods}

\subsection{Study Area}

Our study area is Punjab, India (Figure 1). The state covers an area of 50,362 square kilometers, $1.53 \%$ of India's total geographical area. It is the $20^{\text {th }}$-largest Indian state by area. According to 2011 census of India, total population of Punjab was $27,704,236$. It is the $16^{\text {th }}$ largest state by population, comprising 22 districts.

\subsection{Study Design}

This is retrospective review study carried out to determine trend of 7-year (2012-2018) malaria, dengue and chikungunya cases.

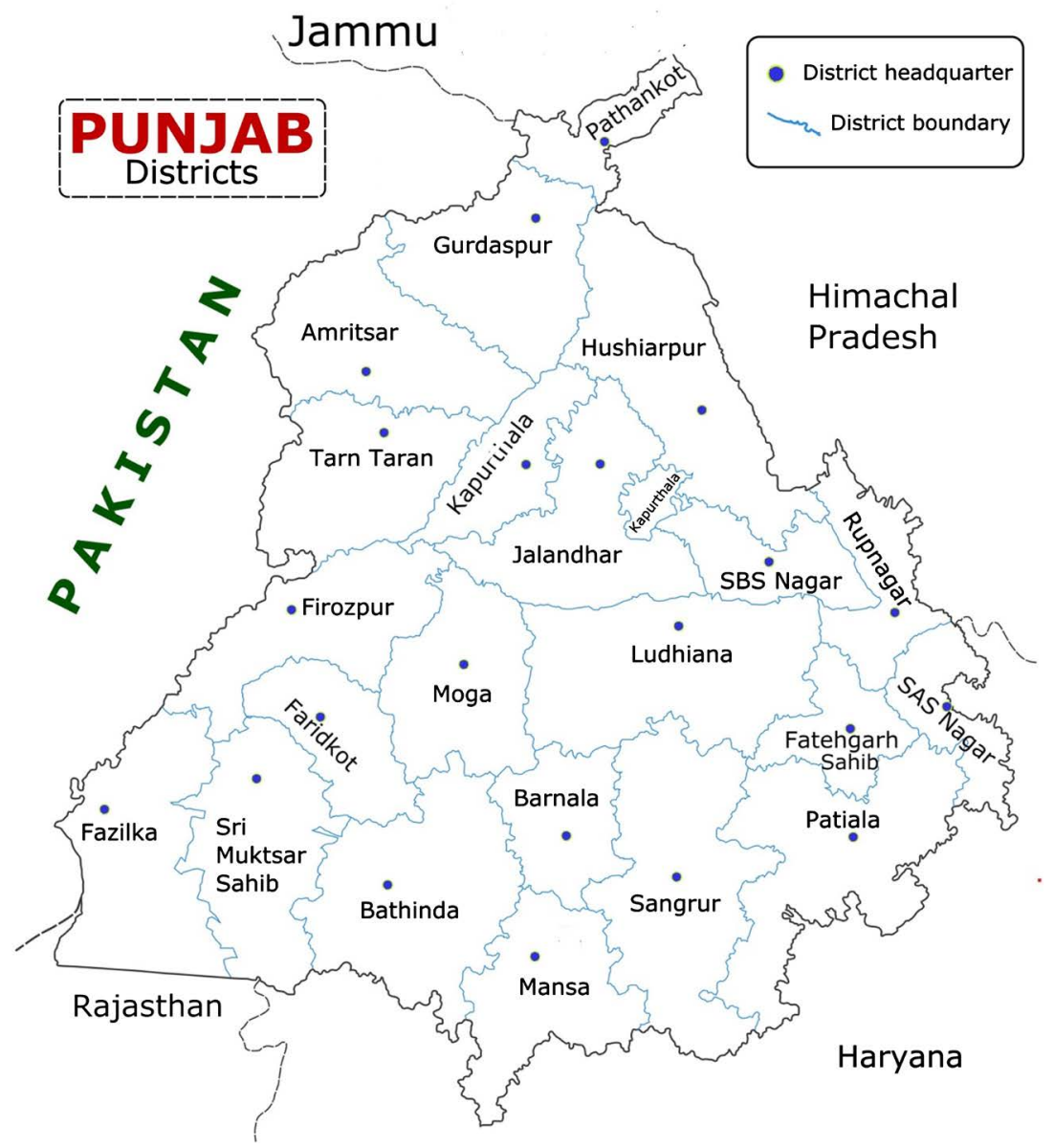

Figure 1. Map showing study areas of Punjab, India. 


\subsection{Data Collection}

Quantitatively retrospective data was collected from Department of Health and Family Welfare, NVBDCP, Punjab from 2012 to 2018. The NVBDCP, Punjab collects epidemiological data from both public and private health facilities in the state.

\subsection{Statistical Analysis}

The final result values were taken as mean with standard deviation (mean \pm S.D.). Normality for data was examined by the Shapiro-Wilk test. GraphPad Prism 6 for windows version 6.01 (GraphPad Software, Inc., San Diego Calfornia, USA) was utilized to perform the statistical analysis. Kruskall Wallis test followed by post hoc Tukey test was used to calculate statistical significance between groups. Statistical significance values of less than $0.05\left({ }^{*} \mathrm{p}<0.05,{ }^{* *} \mathrm{p}<0.01,{ }^{* * *} \mathrm{p}<0.001\right.$ and $\left.{ }^{* * * *} \mathrm{p}<0.0001\right)$ were considered to be significant in all cases.

\section{Results}

\subsection{Annual Prevalence of Cases of Malaria, Dengue and Chikungunya}

\section{Malaria}

During the study period (from Jan. 2012 to Dec. 31, 2018) there were 7193 confirmed malaria cases in the 22 districts of Punjab. The highest number of cases $\mathrm{n}=1760$ were recorded in the year 2013. On the other hand, the least number of cases $\mathrm{n}=596$ were recorded in the year 2015 (Figure 2(a), Figure 3(a)). With regard to Plasmodium species, most malaria cases were $P$. vivax $98.15 \%$ (7060) while $1.8 \%$ (130) were $P$. falciparum and $0.04 \%$ (3) were mixed infections ( $P$. falciparum plus $P$. vivax) (Figure 3(b)).

\section{Dengue}

A total of 58,729 dengue cases were recorded in Punjab during the years 2012-2018. The number of dengue cases significantly decreased in 2014, and then increased from 2015 to 2018, showing peak in 2017. The number of dengue cases in 2017 was found to be highest in the last six years in Punjab (Figure 2(a), Figure $3(\mathrm{c})$ ). A total of 85 deaths were reported in last six years with maximum deaths recorded in year 2013 (25) and 2015 (28), respectively (Figure 2(b)).
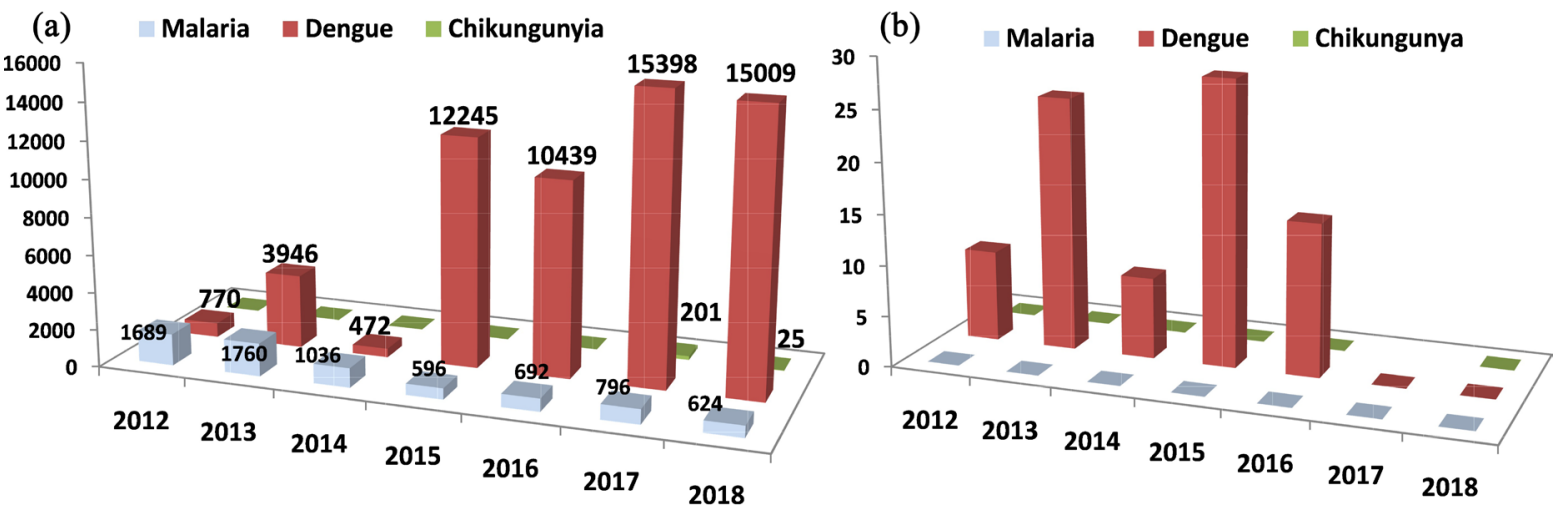

Figure 2. Trend of three major vector borne diseases in Punjab (a) cases and (b) deaths from 2012 to 2018. 

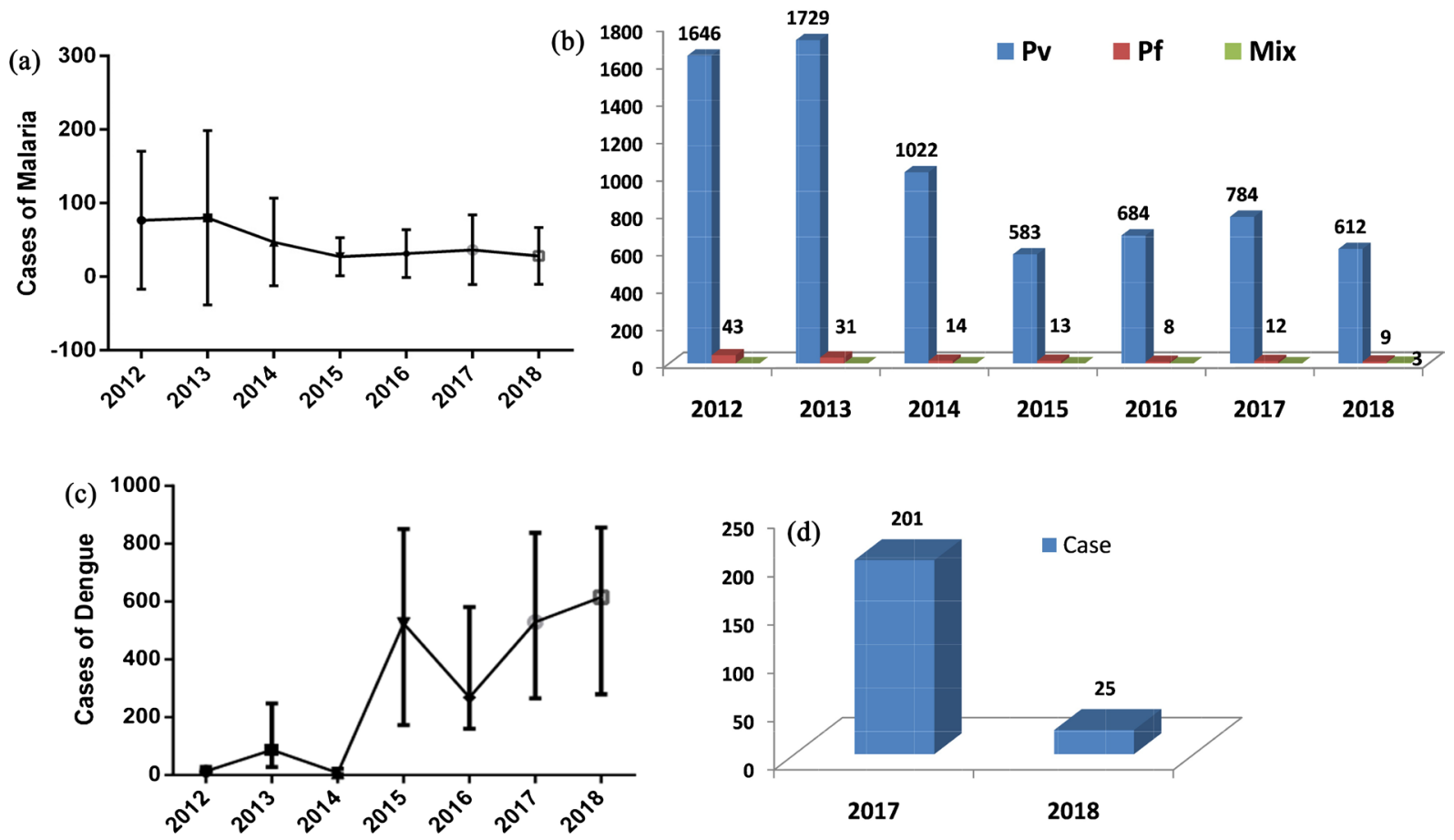

Figure 3. Year wise trend of three major mosquito borne diseases in Punjab, India from 2012 to 2018. (a) Year wise trend of malaria in Punjab; (b) Year wise trend of P. vivax, P. falciparum and mixed infection in Punjab; (c) Year wise trend of dengue in Punjab; (d) Year wise trend of chikungunya.

\section{Chikungunya}

From 2012-2018, a total of 226 cases of chikungunya has been recorded in Punjab. The cases were observed in 2017 and 2018 only, with maximum number in year 2017 (201) (Figure 2(a)).

\subsection{Gender Wise Distribution of Cases of Malaria, Dengue and Chikungunya}

\section{Malaria}

Prevalence of malaria infections was higher in males $4394 / 7193$ (61\%) than females 2799 (38.9\%). Malaria prevalence among male individuals was 1.5 times higher than in female individuals over the course of the study showing peak in 2013 (Figure 4(a)).

\section{Dengue}

Of the total dengue cases, 36,397 were male and 21,182 were female. Numbers of male cases were higher than females every year. Highest number of male cases i.e. 9556 was reported in year 2018, but highest number of female cases (5903) was reported in 2017. However least cases of both males and females (306 and 166, respectively) were reported in year 2014 (Figure 4(b)). The largest ratio of 2.22 was observed in year 2012 while as the smallest ratio of 1.61 was observed in year 2017.

\section{Chikungunya}

In 2017, more male cases (110) were observed than female cases (91) whereas in 2018 almost same number of male and female cases was found (Figure 4(c)). 


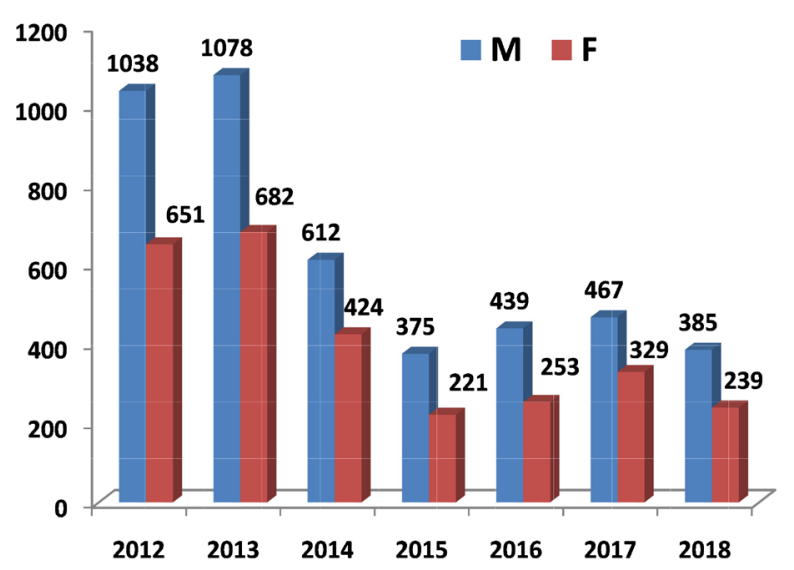

(a)

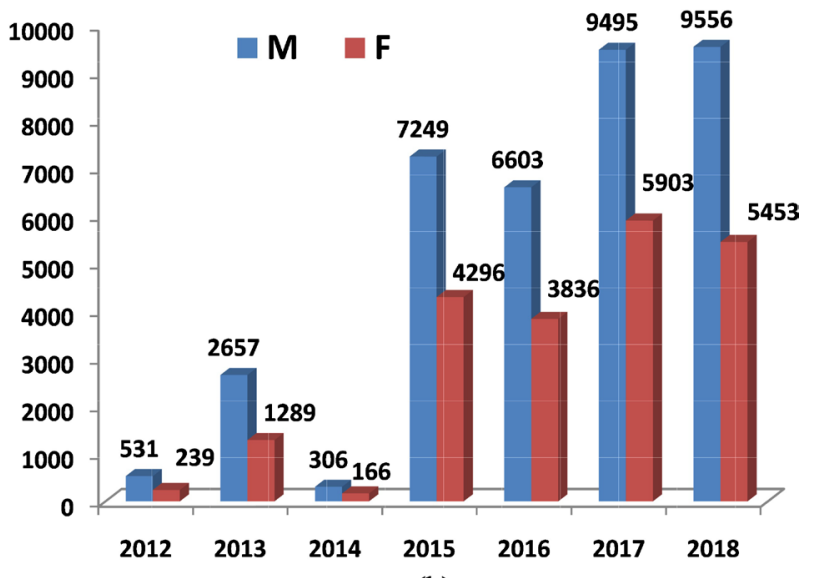

(b)

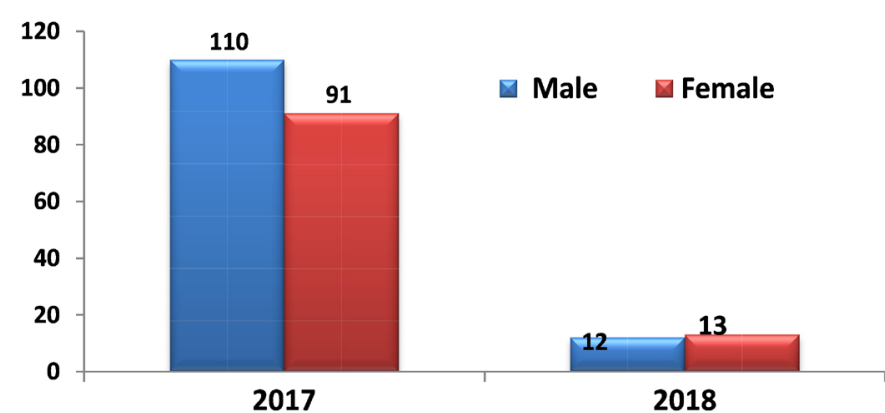

(c)

Figure 4. Gender wise distribution of three major mosquito borne diseases in Punjab from 2012 to 2018. (a) Gender wise cases of malaria; (b) Gender wise cases of dengue and (c) Gender wise cases of chikungunya.

\subsection{District and Area Wise Distribution of Cases of Malaria, Dengue and Chikungunya}

\section{Malaria}

Out of 7193 total positive cases in the 7-year study period in Punjab, 7060 cases were of $P$. vivax. With regard to district, the mean malaria cases were significantly high in Mansa (211.6 \pm 7.29$)$ (Figure 5(a)) throughout seven year trend which indicates it to be endemic district for malaria whereas the lowest mean malaria cases were reported from Fatehgarh Sahib $(4.2 \pm 8.92)$. There was a statistically significant variation $(p<0.0001)$ in malaria prevalence among these districts. With regard to areas, more cases of malaria were reported from rural areas as compared to urban areas (Figure 6(a)).

\section{Dengue}

The maximum number of dengue has been recorded in Patiala, S.A.S. Nagar and Ludhiana for the consecutive period of four years of 2014 to 2018 (Figure 5(b)). With regard to districts, Patiala hold the highest (4357 \pm 8855.3$)$ dengue prevalence in last seven years followed by Ludhiana ( $804.6 \pm 594.8)$ and S.A.S. Nagar $(928.3 \pm 996.6)$ while the lowest dengue cases were reported from district Tarantaran $(64.29 \pm 62.18)$. There was a statistically significant variation ( $\mathrm{p}<$ $0.0001)$ in dengue prevalence among the districts. With respect to areas, more cases of dengue were found in urban areas as compared to rural areas (Figure 6(b)). 

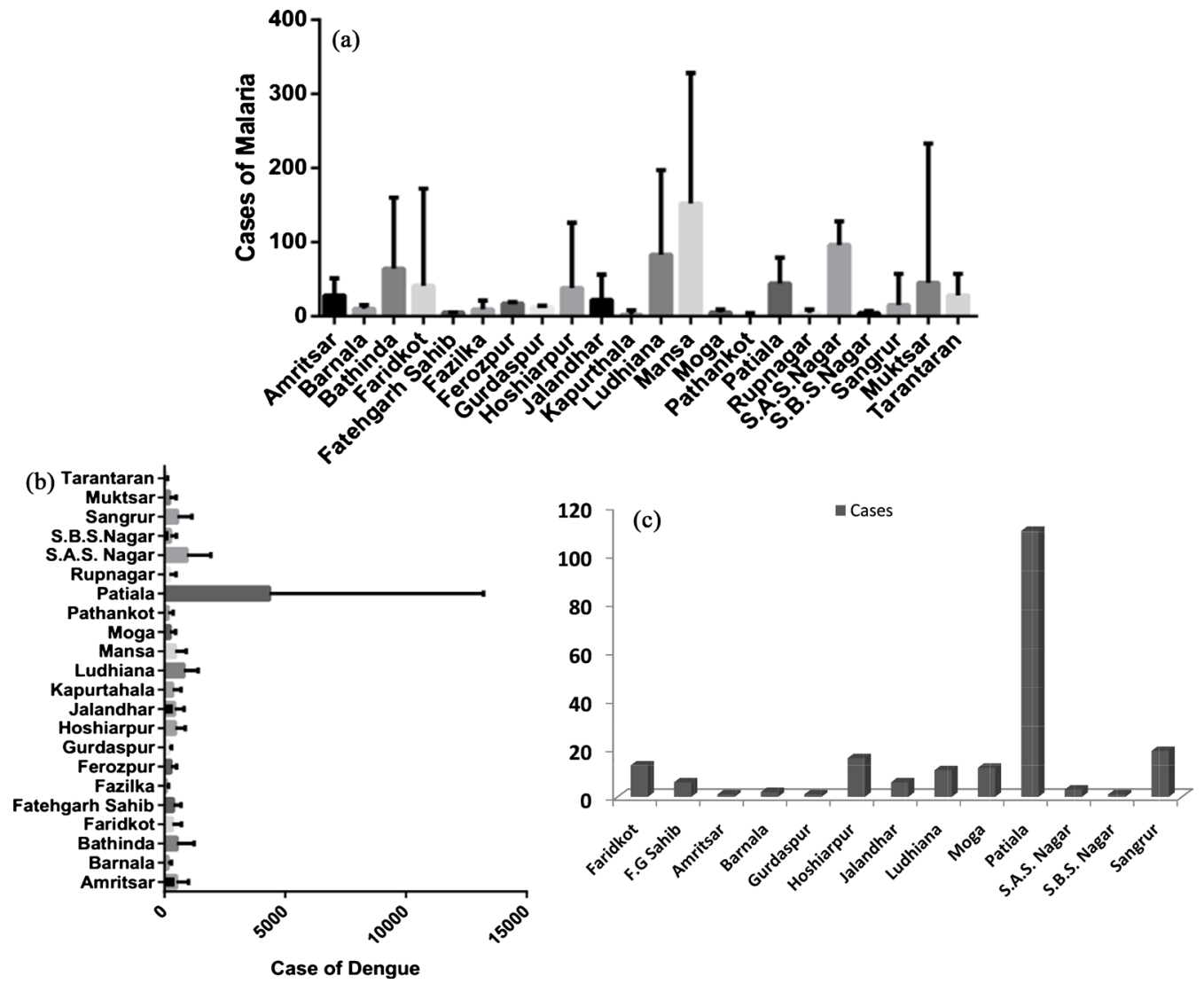

Figure 5. Seven year district wise distribution of three major mosquito borne diseases in Punjab. (a) Malaria; (b) Dengue and (c) Chikungunya.

(a)
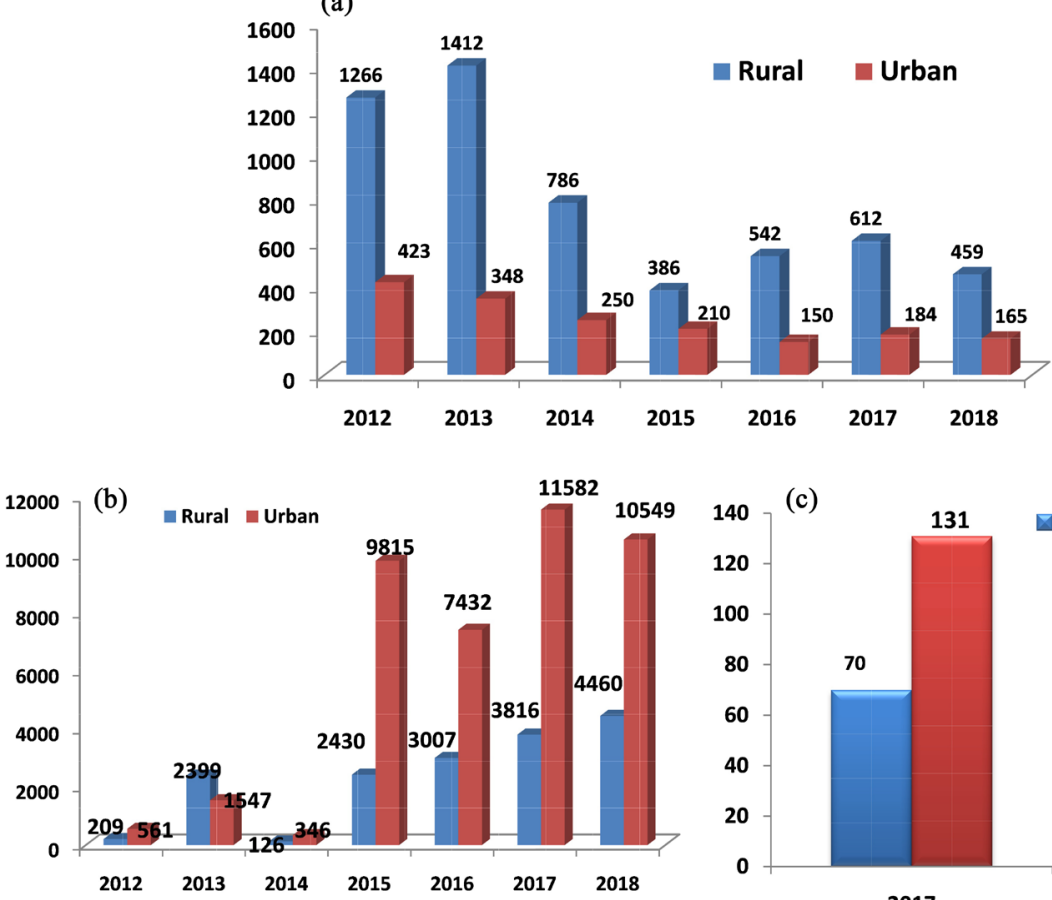

Q Rural U Urban

2017

2018

Figure 6. Area wise distribution of three major mosquito borne diseases in Punjab. (a) Malaria; (b) Dengue and (c) Chikungunya. 


\section{Chikungunya}

In year 2017, maximum chikungunya cases were found in Patiala followed by Sangrur and Hoshiarpur while least number of cases was recorded in Amritsar and Gurdaspur (Figure 5(c)). In both the years 2017 and 2018, more cases were found in urban as compared to rural areas (Figure 6(c)).

\subsection{Seasonal Trend of Cases of Malaria, Dengue, Chikungunya}

\section{Malaria}

In the present study, the prevalence of malaria cases was observed to have increased from June to September with Mean \pm S.D. varying from $100.1 \pm 18.4$ to $324.0 \pm 124.8$ (Figure 7(a)). This period is considered as the peak malaria transmission period in Punjab after rain in July and August with highest cases in month of September compared to rest of months with significant $\mathrm{p}$ value $<0.0001$.

\section{Dengue}

Reporting of dengue positive cases remained low during the first 6 months of a year (January to June) followed by a significant increase from July to November and again start decreasing during December (Figure 7(b)). Therefore, most dengue cases were reported between August and November, especially in October $(27,221)$ and November $(19,173)$ showing significant difference with first six months of year with $\mathrm{p}$ value $<0.0001$ (Figure $7(\mathrm{~b})$ ).

\section{Chikungunya}

The prevalence of chikungunya found to increase from August to October showing highest number of cases in October (Figure 7(c)).
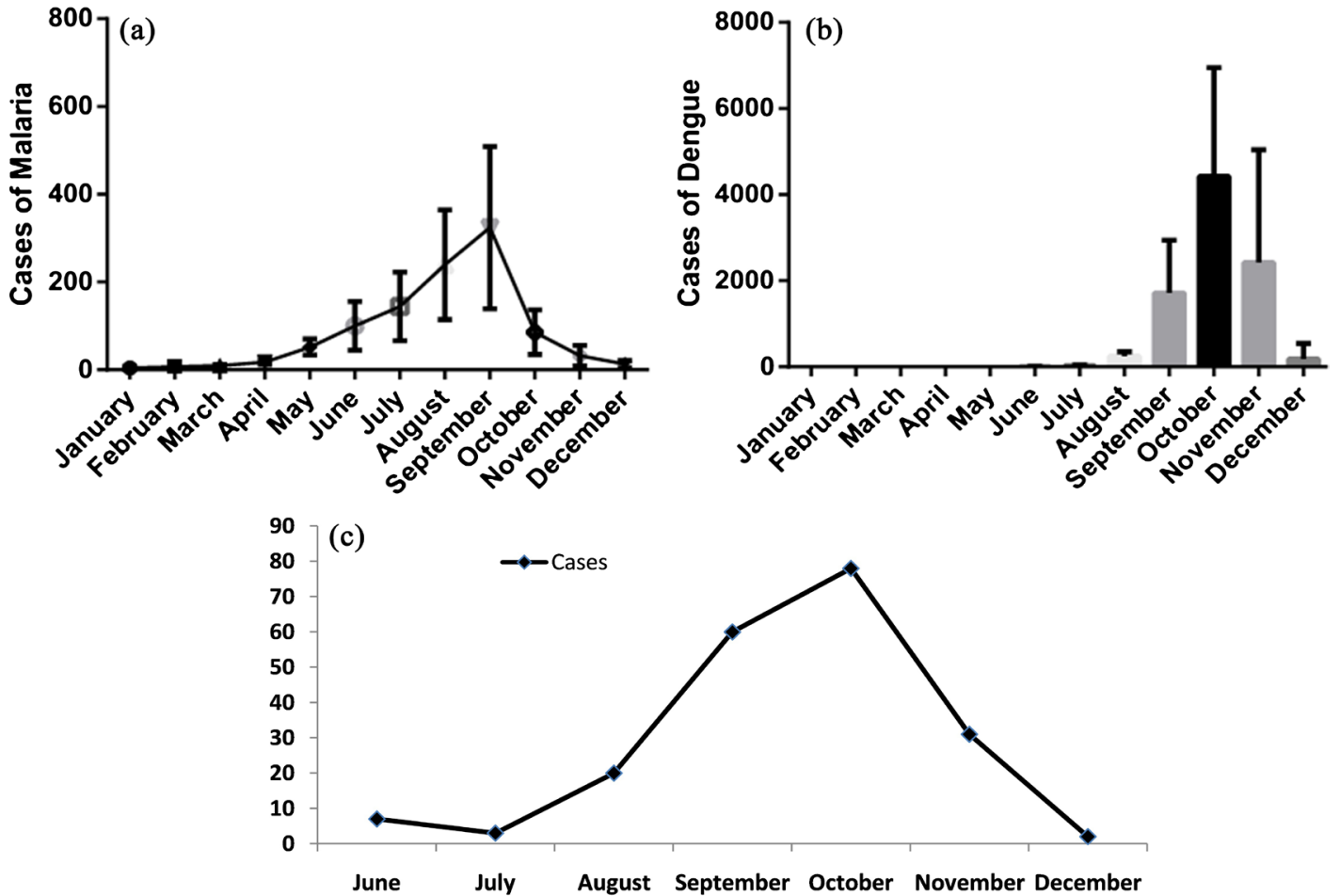

Figure 7. Seasonal prevalence of three major mosquito borne diseases in Punjab. (a) Malaria; (b) Dengue and (c) Chikungunya. 


\section{Discussion}

In Punjab three major mosquito borne diseases viz. Malaria, Dengue and Chikungunya are gradually spreading all over the state. This paper describes the distribution of these vector borne diseases throughout the state and their burden for the past seven years. To our knowledge, this is first ever study from Punjab, India to explain the variation in the burden of the malaria, dengue and chikungunya. From the analysis of quantitative data, the present study showed that in last seven years a total of 7193 confirmed malaria cases were recorded in Punjab with the highest number of cases $\mathrm{n}=1760$ in the year 2013. The state showed high prevalence of $P$. vivax (98.15\%) as compared to $P$. falciparum (1.8\%). For dengue, a total of 58,729 dengue cases were recorded in Punjab during the year 2012-2018 and 2017 to be the highest contributor and cases of chikungunya were recorded only two years 2017 and 2018 during the last seven years.

India has launched the malaria elimination drive and set the goal of malaria elimination by 2030 and the state of Punjab which is reporting $<1$ annual parasite incidence (API) in all the 22 districts for the last five years has qualified for malaria elimination under category-1 as per National Framework of Malaria Elimination 2016-30. Therefore, this trend analysis will be helpful for planning and execution of vector control strategies on priority in endemic districts of Punjab. Though very few studies are published regarding epidemiology of malaria in Punjab, India. One of the data was published regarding epidemiological profile of malaria cases in Amritsar district in years 2015 and 2016 [5], the maximum cases were reported in September and majority of cases were males. In another one year study conducted at Jiwan Jyoti Christian Hospital in Sonbhadra district, Ludhiana, the prevalence of $P$. vivax cases were more as compared to $P$. falciparum [6] which positively correlates with our six year trend analysis of malaria cases in Punjab, India.

Dengue is emerging as the most prevalent mosquito borne viral infection among tropical and subtropical countries and being top most contributor among the three major mosquito borne diseases in Punjab. In one of the few studies published on dengue in Punjab [7] in Tertiary Care Hospital Amritsar, Punjab. In the present study the percentage positivity of Dengue infection amongst the screened population of clinically suspected patients was 100/282. In another study published [8], the objective of this study is to find the clinical profile of Dengue patients treated in a tertiary care hospital in the district Jalandhar of North India. Out of total 376 dengue cases, 206 (54.8\%) were male and 170 (45.2\%) female. 74.7\% patients were urban and $25.3 \%$ rural, statistically significant $(\mathrm{p}<0.001)$. In the present six year trend analysis in different districts of Punjab, males were more affected as compared to females and more cases were from urban areas. These three vector borne diseases are major health problem in India [9]. All three are endemic in many parts of our country and pose enormous burden to the health system.

Besides entomological surveillance, there are lot of determinant factors which influences the epidemiological trend of mosquito borne diseases viz. malaria, dengue and chikungunya. These factors include non-climatic: 1) demographic 
changes like population growth, economical trends and land use patterns 2) global travel and rapid urbanization, rural to urban migration and 3) modern transportation with increased movement of people, commodities, animals, vectors and pathogens whereas climatic factors include environmental variables such as geographical, climatological and hydrological [10]. One of the study showed that due to poor water and sanitation conditions inhabitants have to store water in open containers which act as breeding habitats for the mosquitoes [11] [12]. Moreover, the impact of socio-economic risk factors in malaria spread was highlighted [13] and a socio-economic status index (SES) was constructed. Moreover, socio-economic variables to produce dengue risk maps so as to identify the geographical areas where the risk of a disease outbreak is higher and also studied demographic data to estimate the rate of affected population was studied [14].

Therefore, in coordination with these findings it also necessitates further in-depth evaluation of all other factors which effect epidemiological trend of mosquito borne diseases like geographical, climatic and other demographic factors in the endemic districts of Punjab which were not included in the present study will boost the active entomological surveillance in order to control these three major mosquito borne diseases.

\section{Conclusion}

The results clearly show the declining trend of malaria prevalence in Punjab which indicates the existence of significant malaria control and well developed prevention measures but a great challenge to achieve success in ongoing malaria elimination programme is the existence of high prevalence $P$. vivax in the few districts of Punjab. Therefore, concerned bodies have to act aggressively in order to control the prevalence of $P$. vivax. Moreover, in the present state Dengue remains as a public health problem with increasing incidence rate every year. The results suggest that some districts of Punjab need more attention and emphasis should be on education of the public and prevention in line with achieving the national objectives of reducing dengue burden.

\section{Acknowledgements}

We would like to thank all the Data collectors at respective districts and Department of Health and Family Welfare, Punjab.

\section{Conflicts of Interest}

The authors declare no conflicts of interest regarding the publication of this paper.

\section{References}

[1] WHO (2017) WHO/Vector-Borne Diseases. http://www.who.int/mediacentre/factsheets/fs387/en/.

[2] Bhatt, S., Gething, P.W., Brady, O.J., Messina, J.P., Farlow, A.W., Moyes, C.L., Drake, J.M., Brownstein, J.S., Hoen, A.G., Sankoh, O., Myers, M.F., George, D.B., 
Jaenisch, T., Wint, G.R., Simmons, C.P., Scott, T.W., Farrar, J.J. and Hay, S.I. (2013) The global Distribution and Burden of Dengue. Nature, 25, 504-507. https://doi.org/10.1038/nature12060

[3] Mourya, D.T., Thakare, J.P., Gokhale, M.D., Powers, A.M., Hundekar, S.L., Jaykumar, P.C., Bondre, V.P., Souche, Y.S. and Padbidri, V.S. (2001) Isolation of Chikungunya Virus from Aedes aegypti Mosquitoes Collected in the Town of Yawat, Pune District, Maharashtra State, India. Acta Virology, 45, 305-309.

[4] Lum, F.M., Teo, T.H., Lee, W.W.L., Kam, Y.W., Rénia, L. and Ng, L.F.P. (2013) An Essential Role of Antibodies in the Control of Chikungunya Virus Infection. Journal of Immunology, 190, 6295-6302. https://doi.org/10.4049/jimmunol.1300304

[5] Mahajan, S.L. (2017) Epidemiological Profile of Malaria in District Amritsar in Years 2015 and 2016. Journal of Community Medicine and Public Health, 1, 102. https://doi.org/10.29011/2577-2228.100002

[6] Koshy, J.M. and Koshy, J. (2014) Clinical Profile of Cerebral Malaria at a Secondary Care Hospital. Journal of Family Medicine and Primary Care, 3, 54-57. https://doi.org/10.4103/2249-4863.130276

[7] Malik, N.M., Devi, B. and Oberoi, L. (2019) Prevalence of Dengue Fever and Comparative Analysis of Primary and Secondary Dengue Cases in a Tertiary Care Hospital, Amritsar, Punjab. Global Journal for Research Analysis, 8, 45-47.

[8] Vij, A.S., Joshi, H., Singh, H., Chopra, S., Shivya, Vij, K.K., Kaur, S., Kaur, N., Kaur, A. and Singh, S. (2016) Clinical Study of Dengue Fever in Jalandhar, North India. Scholar Journal of Applied and Medical Science, 4, 4004-4009.

[9] Kumar, P.S.S., Arjun, M.C., Gupta, S.K. and Nongkynrih, B. (2018) Malaria, Dengue and Chikungunya in INDIA-An Update. Indian Journal of Medical Specialities, 9, 25-29. https://doi.org/10.1016/j.injms.2017.12.001

[10] Ferreira, G.L.C. (2012) Global Dengue Epidemiological Trends. Revista do Institute de Medicina Tropical Sao Paulo, 54, S5-S6. https://doi.org/10.1590/S0036-46652012000700003

[11] Quintero, J., Carrasquilla, G., Suárez, R., González, C. and Olano, V.A. (2009) An Ecosystemic Approach to Evaluating Ecological, Socioeconomic and Group Dynamics Affecting the Prevalence of Aedes aegypti in Two Colombian Towns. Cadernos de Saúde Pública, 25, 93-103. https://doi.org/10.1590/S0102-311X2009001300009

[12] Quintero, J., Brochero, H., Manrique-Saide, P., Barrera-Pérez, M., Basso, C., Romero, S., Caprara, A., De Lima Cunha, J.C., Beltrán-Ayala, E., Mitchell-Foster, K., et al. (2014) Ecological, Biological and Social Dimensions of Dengue Vector Breeding in Five Urban Settings of Latin America: A multi-Country Study. BMC Infectious Disease, 14, Article number: 38. https://doi.org/10.1186/1471-2334-14-38

[13] Homan, T., Maire, N., Hiscox, A., Di Pasquale, A., Kiche, I., Onoka, K., Mweresa, C., Mukabana, W.R., Ross, A., Smith, T.A., et al. (2016) Spatially Variable Risk Factors for Malaria in a Geographically Heterogeneous Landscape, Western Kenya: An Explorative Study. Malaria Journal, 15, Article number: 1.

https://doi.org/10.1186/s12936-015-1044-1

[14] Ayala, R.G. and Estrugo, A. (2014) Assessing the Effects of Climate and Socioeconomic Factors on Vulnerability to Vector-Borne Diseases in Latin America. Inter-American Development Bank, Washington, DC, USA.

https://doi.org/10.2139/ssrn.2533567 